\title{
A generalization of Lepage forms in mechanics
}

\author{
J. Šeděnková \\ Department of Mathematics, Tomas Bata University, Zlín, Czech Republic
}

\begin{abstract}
In this paper we generalize the concept of a Lepage form, introduced by Krupka, to forms of arbitrary degree in mechanics. These forms allow us to find a suitable representation of the classes of forms, appearing in variational sequences in mechanics. The structure of Lepage 2-forms is discussed in detail. The Lepage equivalents of the dynamical forms are mentioned.
\end{abstract}

Key words. Lepage form, Euler-Lagrange form, variational sequence.

PACS. 02.30.Xx, 02.40.Vh, 02.40.Yy.

MSC. 35A15, 58A10, 58A20, 70G75.

\section{INTRODUCTION}

In this paper, a construction is introduced, allowing us to generalize the concept of a Lepage form (Krupka $[9,10])$ to forms of arbitrary degree in the higher order variational sequences on fibered manifolds over one-dimensional bases (i.e., in mechanics).

The $r$-th order variational sequence is by definition the quotient sequence of the De Rham sequence on the $r$-jet prolongation of a fibered manifold, factored through its contact subsequence (Krupka [12]). Basic general properties of the sequence, and in particular, of the variational terms (lagrangians, Euler-Lagrange forms and HelmholtzSonin forms) have been studied by several authors. A complete local representation of the $r$-th order variational sequence in mechanics was found by Štefánek [19]. Another representation of all classes in the first order variational sequence was given by Krupka [11]. Musilová and Krbek [16] found a representation of the variational terms in higher order variational sequence in mechanics. Kašparová [6] found a representation of classes of $n$-forms, $(n+1)$-forms and $(n+2)$-forms of the variational sequence in the first order field theory. Her results were extended to the general order by Krbek, Musilová and Kašparová [8]. The representation of all terms in the $r$-th order field theory were found by Krbek, Musilová [7] by the use of a finite version of Anderson's interior Euler operator [1].

Francaviglia, Palese and Vitolo discussed, among others, such questions as the correspondence of variational sequences and bicomplexes, and their relations to spectral sequences $([4,5,20])$.

The need of global concepts in higher order variational theory led to the introduction of the so called Lepage $n$-forms in field theory, and Lepage equivalents of lagrangians. The main idea, going back to Lepage and Dedecker, was that there should exist a 
close connection between the Euler-Lagrange mapping and the exterior derivative of forms (Krupka [10]). Later, the concept of the Lepage form was extended to 2-forms in mechanics and to $(n+1)$-forms in field theory (Krupková $[14,13])$; the Lepage forms have been introduced as closed counterparts of the Euler-Lagrange forms. In [14], the Lepage forms have been applied to the inverse problem in higher order mechanics, and to the order reducibility problem.

In our generalization of Lepage forms we use a slight (finite order) modification of an operator $\mathscr{I}$, acting on forms on jet manifolds, given by Anderson [1] for the case of the variational bicomplex and called by Anderson the interior Euler operator. This operator was already used, and denoted by different symbols, by Kuperschmidt [15], Dedecker and Tulczyjew [3], and Bauderon [2].

\section{VARIATIONAL SEQUENCE}

Let $\pi: Y \rightarrow X$ be a fibered manifold with fibered coordinate systems $(V, \psi), \psi=$ $\left(t, q^{\sigma}\right)$, on $Y$ and $(U, \varphi), \varphi=(t)$ on $X, \operatorname{dim} X=1, \operatorname{dim} Y=m+1$. Denote by $\pi^{r}:$ $J^{r} Y \rightarrow X$ or just $J^{r} Y$ the $r$-jet prolongation of the fibered manifold $\pi: Y \rightarrow X$, the coordinate system is $\left(V^{r}, \psi^{r}\right), \psi^{r}=\left(t, q^{\sigma}, q_{1}^{\sigma}, \cdots, q_{r}^{\sigma}\right)$ on $J^{r} Y$. For small $r$ we denote $q_{0}^{\sigma}=q^{\sigma}, q_{1}^{\sigma}=\dot{q}^{\sigma}, q_{2}^{\sigma}=\ddot{q}^{\sigma}$. The canonical jet projections are $\pi^{r, s}: J^{r} Y \rightarrow J^{s} Y, r>s$ and $\pi^{r, 0}: J^{r} Y \rightarrow Y$.

A differential $k$-form $\rho$ on $J^{r} Y$ is called contact, if it vanishes along the $r$-jet prolongation $J^{r} \gamma$ of every section $\gamma$ of $\pi$.

If $(V, \psi), \psi=\left(t, q^{\sigma}\right)$, is a fibered chart on $Y$, then we often use the contact basis $d t, \omega^{\sigma}, \omega_{1}^{\sigma}, \ldots, \omega_{r}^{\sigma}, d q_{r+1}^{\sigma}$ on $V^{r+1}=\left(\pi^{r+1,0}\right)^{-1} V$ given by the forms

$$
\omega_{j}^{\sigma}=d q_{j}^{\sigma}-q_{j+1}^{\sigma} d t, \quad 0 \leq j \leq r .
$$

Recall that a form which contains exactly $k$ expressions (1) is called $k$-contact. Every form $\rho$ on $J^{r} Y$ can be uniquely decomposed, after the lifting to $J^{r+1} Y$, as the sum of the $k$-contact components $p_{k} \rho$.

Let $\Omega_{k}^{r}$ be the direct image of the sheaf of smooth $k$-forms over $J^{r} Y$ by the jet projection $\pi^{r, 0}$, where $k \geq 0$. Denote

$$
\Omega_{0, c}^{r}=\{0\}, \quad \Omega_{k, c}^{r}=\operatorname{ker} p_{k-1}, \quad \Theta_{k}^{r}=\Omega_{k, c}^{r}+d \Omega_{k-1, c}^{r},
$$

where $k \geq 1$, and $d \Omega_{k-1, c}^{r}$ is the image sheaf of $\Omega_{k-1, c}^{r}$ by $d$. Then for every open set $V \subset Y, \Omega_{k}^{r} V$ (resp. $\Omega_{k, c}^{r} V$ ) is the Abelian group of $k$-forms (resp. $k$-contact $k$-forms) on $V^{r}=\left(\pi^{r, 0}\right)^{-1}(V), d \Omega_{k-1, c}^{r} V$ is the Abelian group of forms which can be locally expressed as differentials of $(k-1)$-contact $(k-1)$-forms on $V^{r}$, and $\Theta_{k}^{r} V$ is a subgroup of $\Omega_{k}^{r} V$. We get a sequence

$$
0 \rightarrow \Theta_{1}^{r} \rightarrow \Theta_{2}^{r} \rightarrow \Theta_{3}^{r} \rightarrow \ldots \rightarrow \Theta_{M}^{r} \rightarrow 0
$$

in which all arrows denote the exterior differentiation $d$, and $M=m r+1$. Sequence (3) is a subsequence of the De Rham sequence

$$
0 \rightarrow \mathbb{R}_{Y} \rightarrow \Omega_{0}^{r} \rightarrow \Omega_{1}^{r} \rightarrow \Omega_{2}^{r} \rightarrow \ldots \rightarrow \Omega_{N-1}^{r} \rightarrow \Omega_{N}^{r} \rightarrow 0,
$$


where $N=\operatorname{dim} J^{r} Y=1+m(r+1)$. The quotient sequence

$$
\begin{gathered}
\quad \rightarrow \mathbb{R}_{Y} \rightarrow \Omega_{0}^{r} \rightarrow \Omega_{1}^{r} / \Theta_{1}^{r} \rightarrow \Omega_{2}^{r} / \Theta_{2}^{r} \rightarrow \ldots \\
\ldots \rightarrow \Omega_{M}^{r} / \Theta_{M}^{r} \rightarrow \Omega_{M+1}^{r} \rightarrow \ldots \rightarrow \Omega_{N-1}^{r} \rightarrow \Omega_{N}^{r} \rightarrow 0
\end{gathered}
$$

is also exact. Sequence (5) is called the $r$-th order variational sequence. The class of a differential form $\rho \in \Omega_{k}^{r} V$ in the variational sequence (5) is denoted by $[\rho]$.

The quotient mapping $E: \Omega_{k}^{r} / \Theta_{k}^{r} \rightarrow \Omega_{k+1}^{r} / \Theta_{k+1}^{r}$ is defined by

$$
E([\rho])=[d \rho]
$$

This mapping satisfies the condition $E^{2}=0$. The quotient mapping $E: \Omega_{1}^{r} / \Theta_{1}^{r} \rightarrow \Omega_{2}^{r} / \Theta_{2}^{r}$ is called the Euler-Lagrange mapping. The quotient mapping $E: \Omega_{2}^{r} / \Theta_{2}^{r} \rightarrow \Omega_{3}^{r} / \Theta_{3}^{r}$ is called the Helmholtz-Sonin mapping.

A lagrangian of order $r$ is a $\pi^{r}$-horizontal $n$-form $\lambda$. In coordinates, the following can be written

$$
\lambda=L d t
$$

where $L$ is a function on $J^{r} Y$ called Lagrange function.

Let $\rho$ be a 1 -form on $J^{r} Y$. A form $\rho$ is called a Lepage 1 -form if $p_{1} d \rho$ is a $\pi^{r+1,0_{-}}$ horizontal 2-form. A Lepage form $\rho$ is called a Lepage equivalent of a lagrangian $\lambda$ if $h \rho=\lambda$. It is known that in higher order mechanics, Lepage equivalents are uniquely determined by lagrangians. We denote by $\theta_{\lambda}$ the Lepage equivalent of a lagrangian $\lambda$. If $r=1, \theta_{\lambda}$ is the well known Poincaré-Cartan form, if $r>1$, we have the generalized Poincaré-Cartan form. If in a fibered chart $\lambda=L d t$, then

$$
p_{1} d \theta_{\lambda}=E_{\sigma}(L) \omega^{\sigma} \wedge d t
$$

where

$$
E_{\sigma}(L)=\sum_{l=0}^{r}(-1)^{l} \frac{d^{l}}{d t^{l}} \frac{\partial L}{\partial q_{l}^{\sigma}} .
$$

The form (8) is called the Euler-Lagrange form and it is denoted by $E_{\lambda}$. The components (9) are called the Euler-Lagrange expressions.

\section{THE INTERIOR EULER-LAGRANGE OPERATOR}

We recall basic properties of the interior Euler-Lagrange operator rewritten in the form presented in Šeděnková [18].

Let $(V, \psi), \psi=\left(t, q^{\sigma}\right)$, be a fibered chart on $Y$ and let $\left(V^{2 r+1}, \psi^{2 r+1}\right), \psi^{2 r+1}=$ $\left(t, q^{\sigma}, q_{1}^{\sigma}, \ldots, q_{2 r+1}^{\sigma}\right)$, be the associated fibered chart on $J^{2 r+1} Y$. We set

$$
\Xi=\frac{\partial}{\partial t}+\sum_{j=0}^{2 r} q_{j+1}^{\sigma} \frac{\partial}{\partial q_{j}^{\sigma}},
$$


$\Xi$ is a vector field on $V^{2 r+1}$. If $\rho \in \Omega_{k+1}^{r} V, k \geq 1$, we define a form on $V^{2 r+1}$ by

$$
\mathscr{I}_{(V, \psi)}(\rho)=\frac{1}{k} \omega^{\alpha} \wedge \sum_{j=0}^{r}(-1)^{j} \partial_{\Xi}^{j} i_{\frac{\partial}{\partial q_{j}^{\alpha}}} p_{k} \rho
$$

where $\partial_{\Xi}$ is the Lie derivative with respect to the vector field $\Xi, \partial_{\Xi}^{j}$ is the $j$-th power of $\partial_{\Xi}$, and $i_{\partial / \partial q_{j}^{\alpha}}$ denotes the contraction by the vector field $\partial / \partial q_{j}^{\alpha}$. For $k=0$ and $\rho \in \Omega_{1}^{r} V$, we define

$$
\mathscr{I}_{(V, \psi)}(\rho)=h \rho .
$$

Note that the form $\mathscr{I}_{(V, \psi)}(\rho)$ depends only on the $k$-contact $(k+1)$-form $p_{k} \rho$.

The following two lemmas and Theorem 1 can be proved in fibered coordinates.

Lemma 1. Let $\rho \in \Omega_{k+1}^{r} V, k \geq 1$. Then the following equation is satisfied

$$
\frac{1}{k} \omega^{\alpha} \wedge \sum_{j=0}^{r}(-1)^{j} \partial_{\Xi}^{j} i_{\frac{\partial}{\partial q_{j}^{\alpha}}} p_{k} \rho=p_{k} \rho+\frac{1}{k} \sum_{j=1}^{r} \sum_{l=1}^{j}(-1)^{l}\left(\begin{array}{l}
j \\
l
\end{array}\right) \partial_{\Xi}^{l}\left(\omega_{j-l}^{\alpha} \wedge i_{\frac{\partial}{\partial q_{j}^{\alpha}}} p_{k} \rho\right) \text {. }
$$

Lemma 2. Let $(V, \psi), \psi=\left(t, q^{\sigma}\right),(\bar{V}, \bar{\psi}), \bar{\psi}=\left(\bar{t}, \bar{q}^{\sigma}\right)$ be two fibered charts on $Y$ such that $V \cap \bar{V} \neq 0$. Then for every $\rho \in \Omega_{k+1}^{r}(V \cap \bar{V}), k \geq 0$,

$$
\mathscr{I}_{(V, \psi)}(\rho)=\mathscr{I}_{(\bar{V}, \bar{\psi})}(\rho) \text {. }
$$

It follows from Lemma 2 that equations (11) define a global operator $\mathscr{I}: \Omega_{k+1}^{r} \rightarrow$ $\Omega_{k+1}^{2 r+1} . \mathscr{I}$ is called the interior Euler-Lagrange operator. The differential form $\mathscr{I}(\rho)$ is called the canonical representative of a differential form $\rho$. The operator $\mathscr{I}$ generates new sequence

$$
0 \rightarrow \mathbb{R}_{Y} \rightarrow \Omega_{0}^{r} \rightarrow \mathscr{I} \Omega_{1}^{r} \rightarrow \mathscr{I} \Omega_{2}^{r} \rightarrow \ldots \rightarrow \mathscr{I} \Omega_{N-1}^{r} \rightarrow \mathscr{I} \Omega_{N}^{r} \rightarrow 0
$$

which is isomorfic with the variational sequence (5).

The following theorem characterizes properties of $\mathscr{I}$. In particular, it turns out that the kernels of $\mathscr{I}$ coincide with the spaces in the subsequence (3) of the De Rham sequence (4).

Theorem 1. Let $\pi: Y \rightarrow X$ be a fibered manifold over one-dimensional base $X$. Let $k \geq 0$.

(a) For every open set $V \subset Y$ and every $\rho \in \Omega_{k+1}^{r} V, \mathscr{I}(\rho)$ lies in the same class as $\left(\pi^{2 r+1, r}\right)^{*} \rho$.

(b) The operator $\mathscr{I}$ satisfies $\mathscr{I}^{2}=\mathscr{I}$ (up to the canonical projection).

(c) For every open set $V \subset Y$, the kernels of $\mathscr{I}$ coincide with $\Theta_{k+1}^{r} V$. 


\section{LEPAGE FORMS}

Let $k \geq 0$. A form $\rho \in \Omega_{k+1}^{r} V$ is called a Lepage form, if

$$
p_{k+1} d \rho=\mathscr{I}(d \rho) \text {. }
$$

For $k=0$, this definition reduces to the original one (Krupka [9, 10]; for more details we refer to Šeděnková [18]). If $\rho$ is a Lepage form, then the forms $d \rho$ and $\rho+d \eta$, where $\eta$ is arbitrary, are trivially also Lepage forms. The meaning of Lepage forms consists in a generalization of formulas (8), (9); if $k=1$, then $p_{2} d \rho$ is the Helmholtz-Sonin form (compare with Krupka [11] for the first order case).

We now analyze the structure of Lepage 2-forms in higher order mechanics. Because of the lack of space, we restrict ourselves to preliminary results; more details as well as proofs will be given elsewhere.

Theorem 2. Let $\rho \in \Omega_{2}^{1} V$, let in a fibered chart

$$
\rho=a_{\sigma} \omega^{\sigma} \wedge d t+b_{\sigma} d \dot{q}^{\sigma} \wedge d t+c_{\sigma v} \omega^{\sigma} \wedge \omega^{v}+d_{\sigma v} d \dot{q}^{\sigma} \wedge \omega^{v}+e_{\sigma v} d \dot{q}^{\sigma} \wedge d \dot{q}^{v},
$$

the coefficients $c_{\sigma v}, e_{\sigma v}$ are antisymmetric in $\sigma, v$. The following three conditions are equivalent:

(a) $\rho$ is a Lepage form

(b) $\rho$ satisfies

$$
\begin{aligned}
\frac{\partial e_{\sigma v}}{\partial \dot{q}^{\lambda}}+\frac{\partial e_{v \lambda}}{\partial \dot{q}^{\sigma}}+\frac{\partial e_{\lambda \sigma}}{\partial \dot{q}^{v}} & =0, \\
d_{\sigma v}-d_{v \sigma}-\frac{\partial b_{\sigma}}{\partial \dot{q}^{v}}+\frac{\partial b_{v}}{\partial \dot{q}^{\sigma}}+2 \frac{\partial e_{\sigma v}}{\partial t}+2 \frac{\partial e_{\sigma v}}{\partial q^{\lambda}} \dot{q}^{\lambda} & =0, \\
\frac{\partial d_{\sigma v}}{\partial \dot{q}^{\lambda}}-\frac{\partial d_{v \sigma}}{\partial \dot{q}^{\lambda}}+\frac{\partial d_{\lambda \sigma}}{\partial \dot{q}^{v}}-\frac{\partial d_{\lambda v}}{\partial \dot{q}^{\sigma}}+2 \frac{\partial e_{\lambda \sigma}}{\partial q^{v}}-2 \frac{\partial e_{\lambda v}}{\partial q^{\sigma}} & =0, \\
\frac{\partial a_{v}}{\partial \dot{q}^{\sigma}}-\frac{\partial a_{\sigma}}{\partial \dot{q}^{v}}+\frac{\partial b_{v}}{\partial q^{\sigma}}-\frac{\partial b_{\sigma}}{\partial q^{v}}+\frac{\partial d_{\sigma v}}{\partial t}-\frac{\partial d_{v \sigma}}{\partial t} & =0 . \\
+\frac{\partial d_{\sigma v}}{\partial q^{\lambda}} \dot{q}^{\lambda}-\frac{\partial d_{v \sigma}}{\partial q^{\lambda}} \dot{q}^{\lambda}+4 c_{\sigma v} & =0 .
\end{aligned}
$$

(c) There exist functions $A_{\sigma}$, and a 1-form $\eta$ such that

$$
\rho=A_{\sigma} \omega^{\sigma} \wedge d t+\frac{1}{4}\left(\frac{\partial A_{\sigma}}{\partial \dot{q}^{v}}-\frac{\partial A_{v}}{\partial \dot{q}^{\sigma}}\right) \omega^{\sigma} \wedge \omega^{v}+d \eta .
$$

Now we consider second order Lepage 2-forms. We have the following result.

Theorem 3. Let $\rho \in \Omega_{2}^{2} V$, let in a fibered chart

$$
\begin{aligned}
\rho & =a_{\sigma} \omega^{\sigma} \wedge d t+b_{\sigma} \dot{\omega}^{\sigma} \wedge d t+c_{\sigma} d \ddot{q}^{\sigma} \wedge d t+d_{\sigma v} \omega^{\sigma} \wedge \omega^{v}+e_{\sigma v} \dot{\omega}^{\sigma} \wedge \omega^{v} \\
& +f_{\sigma v} \dot{\omega}^{\sigma} \wedge \dot{\omega}^{v}+g_{\sigma v} d \ddot{q}^{\sigma} \wedge \omega^{v}+h_{\sigma v} d \ddot{q}^{\sigma} \wedge \dot{\omega}^{v}+i_{\sigma v} d \ddot{q}^{\sigma} \wedge d \ddot{q}^{v}
\end{aligned}
$$


and

$$
\begin{aligned}
p_{2} d \rho & =P_{\sigma v} \omega^{\sigma} \wedge \omega^{v} \wedge d t+Q_{\sigma v} \dot{\omega}^{\sigma} \wedge \omega^{v} \wedge d t+R_{\sigma v} \ddot{\omega}^{\sigma} \wedge \omega^{v} \wedge d t \\
& +S_{\sigma v} \dot{\omega}^{\sigma} \wedge \dot{\omega}^{v} \wedge d t+T_{\sigma v} \ddot{\omega}^{\sigma} \wedge \dot{\omega}^{v} \wedge d t+U_{\sigma v} \ddot{\omega}^{\sigma} \wedge \ddot{\omega}^{v} \wedge d t,
\end{aligned}
$$

the coefficients $d_{\sigma v}, f_{\sigma v}, i_{\sigma v}, P_{\sigma v}, S_{\sigma v}, U_{\sigma v}$ are antisymmetric in $\sigma, v$. The following three conditions are equivalent:

(a) $\rho$ is a Lepage form

(b) The components of $p_{2} d \rho$ satisfy

$$
\begin{gathered}
U_{\sigma v}-U_{v \sigma}=0, \quad T_{\sigma v}=0, \quad S_{\sigma v}-S_{v \sigma}=0, \\
R_{\sigma v}+R_{v \sigma}=0, \quad Q_{\sigma v}-Q_{v \sigma}-2 \frac{d}{d t} R_{\sigma v}=0 .
\end{gathered}
$$

(c) There exist functions $A_{\sigma}$ satisfying

$$
\frac{\partial}{\partial \ddot{q}^{\tau}}\left(\frac{\partial a_{\sigma}}{\partial \ddot{q}^{v}}-\frac{\partial a_{v}}{\partial \ddot{q}^{\sigma}}\right)=0
$$

and a 1-forn $\eta$ such that

$$
\begin{aligned}
\rho & =a_{\sigma} \omega^{\sigma} \wedge d t+\frac{1}{4}\left(\frac{\partial a_{\sigma}}{\partial \dot{q}^{v}}-\frac{\partial a_{v}}{\partial \dot{q}^{\sigma}}-\frac{d}{d t}\left(\frac{\partial a_{\sigma}}{\partial \ddot{q}^{v}}-\frac{\partial a_{v}}{\partial \ddot{q}^{\sigma}}\right)\right) \omega^{\sigma} \wedge \omega^{v} \\
& -\frac{1}{2}\left(\frac{\partial a_{\sigma}}{\partial \ddot{q}^{v}}+\frac{\partial a_{v}}{\partial \ddot{q}^{\sigma}}\right) \dot{\omega}^{\sigma} \wedge \omega^{v}+d \eta
\end{aligned}
$$

We note that the coefficients in (24) can be expressed in terms of the coefficients in (23); then (25) become conditions for the coefficients of $\rho$.

Analogous results can also be given for $r$-th order 2-forms.

Theorem 4. Let $\rho \in \Omega_{2}^{r} V$, let in a fibered chart

$$
\begin{aligned}
\rho & =\sum_{i=0}^{r-1} a_{\sigma}^{i} \omega_{i}^{\sigma} \wedge d t+b_{\sigma}^{r} d q_{r}^{\sigma} \wedge d t \\
& +\sum_{i, j=0}^{r-1} c_{\sigma v}^{i j} \omega_{i}^{\sigma} \wedge \omega_{j}^{v}+\sum_{j=0}^{r-1} d_{\sigma v}^{r j} d q_{r}^{\sigma} \wedge \omega_{j}^{v}+e_{\sigma \nu}^{r r} d q_{r}^{\sigma} \wedge d q_{r}^{v}
\end{aligned}
$$

and

$$
p_{2} d \rho=\sum_{j=0}^{r} H_{\sigma \nu}^{0 j} \omega^{\sigma} \wedge \omega_{j}^{v} \wedge d t+\sum_{i, j=1}^{r} H_{\sigma \nu}^{i j} \omega_{i}^{\sigma} \wedge \omega_{j}^{v} \wedge d t .
$$

the coefficients $e_{\sigma \nu}^{r r}$ are antisymmetric in $\sigma, v$, the coefficients $c_{\sigma \nu}^{i j}, H_{\sigma \nu}^{i j}$ are antisymmetric in pairs $\left(\begin{array}{c}i \\ \sigma\end{array}\right),\left(\begin{array}{l}j \\ v\end{array}\right)$. Then $\rho$ is Lepage if and only if

$$
\begin{array}{rlrl}
H_{\sigma \nu}^{i j}-H_{\nu \sigma}^{j i} & =0, & & 1 \leq i, j \leq r, \\
H_{\sigma \nu}^{0 j}+(-1)^{j} H_{v \sigma}^{0 j}+\sum_{l=j+1}^{r}(-1)^{l}\left(\begin{array}{l}
l \\
j
\end{array}\right) \frac{d^{l-j}}{d t^{l-j}} H_{v \sigma}^{0 l}=0, & 1 \leq j \leq r .
\end{array}
$$


Finally, we define Lepage equivalents of the canonical representatives of differential forms. Let $\beta \in \Omega_{k+1}^{s} / \Theta_{k+1}^{s}$ be a class, i.e., let $\beta=\mathscr{I}(\eta)$ for some $\eta \in \Omega_{k+1}^{s} V$. A form $\rho \in \Omega_{k+1}^{r} V$ is said to be a Lepage equivalent of $\beta$, if $\rho$ is a Lepage form, and

$$
p_{k} \rho=\beta .
$$

In particular, this definition includes Lepage equivalents of dynamical forms (i.e., the canonical representatives of 2-forms). In particular, let $E=E_{\sigma} \omega^{\sigma} \wedge d t$ be the second order dynamical form with the functions $E_{\sigma}$ linear in coordinates $\ddot{q}^{v}$. Then Lepage equivalent $\rho_{E}$ of the dynamical form $E$ has the form

$$
\begin{aligned}
\rho_{E} & =E_{\sigma} \omega^{\sigma} \wedge d t+\frac{1}{4}\left(\frac{\partial E_{\sigma}}{\partial \dot{q}^{v}}-\frac{\partial E_{v}}{\partial \dot{q}^{\sigma}}-\frac{d}{d t}\left(\frac{\partial E_{\sigma}}{\partial \ddot{q}^{v}}-\frac{\partial E_{v}}{\partial \ddot{q}^{\sigma}}\right)\right) \omega^{\sigma} \wedge \omega^{v} \\
& -\frac{1}{2}\left(\frac{\partial E_{\sigma}}{\partial \ddot{q}^{v}}+\frac{\partial E_{v}}{\partial \ddot{q}^{\sigma}}\right) \dot{\omega}^{\sigma} \wedge \omega^{v}
\end{aligned}
$$

(compare with second order Lepage form (27)).

\section{ACKNOWLEDGMENTS}

I would like to thank Prof. Demeter Krupka for his remarks and comments. I am obliged to Cankaya University for its scholarship which allowed me to present my talk in their conference. I would also like to acknowledge the financial support of the Czech Grant Agency (grant no. 201/03/0512).

\section{REFERENCES}

1. I. M. Anderson, The Variational Bicomplex, Utah State University, Technical Reports, 1989.

2. M. Bauderon, Le probleme inverse du calcul des variations, Ann. Inst. Henri Poincaré, A 36, (1982), 159-179.

3. P. Dedecker, W. M. Tulczyjew, Spectral sequences and the inverse problem of the calculus of variations, Diff. Geom. Methods in Math. Phys., Proc. Conf. Aix-en-Provence and Salamanca 1979, Lect. Notes Math., 836, (1980), 498-503.

4. M. Francaviglia, M. Palese, Second order variations in variational sequences, Coll. on Diff. Geom. Proc. Conf., Debrecen, Hungary, July 2000, 119-130.

5. M. Francaviglia, M. Palese, R. Vitolo, Symmetries in finite order variational sequences, Czech. Math. J., 52, 127, (2002), 197-213.

6. J. Kašparová (Krpcová), A representation of the 1st-order variational sequence in field theory, Diff. Geom. Appl., Satellite Conference of ICM in Berlin, Aug. 10-14, 1998, Brno, Masaryk University in Brno (1999), 493-502.

7. M. Krbek, J. Musilová, Representation of the variational sequence, Rep. Math. Phys., Torun, 2002, 51, (2003), 251-258.

8. M. Krbek, J. Musilová, J. Kašparová, Representation of the variational sequence in field theory, Steps in Diff. Geom., L. Kozma, P. T. Nagy and L. Tamássy, eds., Proc. Coll. on Diff. Geom., Debrecen, Hungary, July 2000 (Univ. Debrecen, Debrecen, 2001), 147-160. 
9. D. Krupka, Lepage forms in higher order variational theory, Modern developments in analytical mechanics, Vol. I, Geometrical Dynamics, Proc. IUTAM-ISIMM Symp., Torino/Italy 1982, (1983), 197-238.

10. D. Krupka, Some Geometric Aspects of Variational Problems in Fibered Manifolds, Folia Fac. Sci. Nat. Univ. Purk. Brunensis, Physica, XIV, Brno, Czechoslovakia, 1973, pp. 65., arXiv: math$\mathrm{ph} / 0110005$.

11. D. Krupka, Variational sequences in mechanics, Calc. Var., 5, (1997), 557-583.

12. D. Krupka, Variational sequences on finite order jet spaces, Diff. Geom. Appl., Proc. Conf., Brno (Czechoslovakia), J. Janyška and D. Krupka, eds., August 1989; World Scientific, Singapore (1990), 236-254.

13. O. Krupková, Hamiltonian field theory revisited: A geometric approach to regularity, L. Kozma, (ed.) et al., Steps in Diff. Geom., Proc. Coll. on Diff. Geom., Debrecen, Hungary, July 25-30, 2000, Debrecen: Univ. Debrecen, Institute of Mathematics and Informatics (2001), 187-207.

14. O. Krupková, Lepage 2-forms in higher order Hamiltonian mechanics I. Regularity, Arch. Math., 22, No. 2, Brno (1986), 97-120.

15. B. A. Kuperschmidt, Geometry of jet bundles and the structure of Lagrangian and Hamiltonian formalisms, Geometric Methods in Math. Phys., Proc. NSF-CBMS Conf., Lowell/Mass. 1979, Lect. Notes Math. 775 (1980), 162-218.

16. J. Musilová, M. Krbek, A note to the representation of the variational sequence in mechanics, Diff. Geom. Appl., I. Kolář, O. Kowalski, D. Krupka. J. Slovák, eds., Proc. Conf., Brno, Czech Republic, August 1998 (Masaryk University, Brno, 1999) 511-523.

17. J. Šeděnková, On the invariant variational sequences in mechanics, The Proceedings of the 22th Winter School Geometry and Physics, Srni, January 2002; Rend. Circ. Mat. Palermo, Ser. II, 71, (2003), 185-190.

18. J. Šeděnková, Representations of variational sequences and Lepage forms, Ph.D. Thesis, Palacky University, Olomouc, 2004.

19. J. Štefánek, A representation of the variational sequence in higher order mechanics, J. Janyska, (ed.) et al., Diff. Geom. Appl., Proc. of the 6th Int. Conf., Brno, Czech Republic, 1995, (Masaryk University, Brno, 1996), 469-478.

20. R. Vitolo, On different geometric formulations of Lagrangian formalism, Diff. Geom. Appl., 10, No. 3, (1999), 225-255. 
Copyright of AIP Conference Proceedings is the property of American Institute of Physics and its content may not be copied or emailed to multiple sites or posted to a listserv without the copyright holder's express written permission. However, users may print, download, or email articles for individual use. 